\title{
GEOGEBRA PARA EL FORTALECIMIENTO DEL PENSAMIENTO ESPACIAL EN CÁLCULO DIFERENCIAL
}

\section{GEOGEBRA FOR THE STRENGTHENING OF SPACE THINKING IN DIFFERENTIAL CALCULUS}

\author{
Mg. Henry Carrascal Carrascal ${ }^{\mathrm{a}}$, Esp. Jorge Ernesto Cháves Peña ${ }^{\mathrm{b}}$, Esp. Malka Irina Cabellos \\ Martínez ${ }^{\mathrm{c}}$ \\ ${ }^{a}$ Universidad Francisco de Paula Santander Ocaña, Grupo de Investigación GIFEAH, \\ Vía Acolsure, Sede Algodonal, Ocaña, Colombia, hcarrascalc@ufpso.edu.co \\ ${ }^{\mathrm{b}}$ Universidad Francisco de Paula Santander Ocaña, Grupo de Investigación GIFEAH, \\ Vía Acolsure, Sede Algodonal, Ocaña, Colombia, jechavesp@ufpso.edu.co \\ ${ }^{\mathrm{c}}$ Universidad Francisco de Paula Santander Ocaña, Grupo de Investigación INGAP, \\ Vía Acolsure, Sede Algodonal, Ocaña, Colombia, micabellosm@ufpso.edu.co
}

Fecha de recepción: 25-04-2017

Fecha de aprobación: 22-06-2017

\begin{abstract}
Resumen: Evidenciadas las debilidades en el proceso de aprendizaje del cálculo diferencial en los estudiantes de Ingeniería en la Universidad Francisco de Paula Santander Ocaña, se buscó organizar un grupo de docentes y estudiantes en una Comunidad de Aprendizaje y un Club de Matemáticas, para el uso de software de aplicación que permitiera el fortalecimiento de la percepción y el pensamiento gráfico, elementos claves para la concepción espacial del entorno. La investigación tuvo un carácter cualitativo pues buscó determinar el nivel de pensamiento espacial de los estudiantes de la línea de cálculo de los programas de ingeniería, y, cuantitativo pues buscó medir el uso de software de aplicación. Concluido el proyecto se observó que los estudiantes mejoraron en la representación gráfica de funciones bidimensionales, a partir de la utilización de Geogebra, pudiendo resolver ejercicios en menos tiempo, además de visualizarlos gráficamente. También les resultó más fácil despejar sus dudas en relación con cualquier tema de cálculo diferencial que se presentaron en el contenido programático de esta área. Lo anterior permitió concluir que el pensamiento espacial de los estudiantes les habilita la capacidad para orientarse en el espacio y lograr resolver los ejercicios que se les presentan en el aula de clase.
\end{abstract}

Palabras clave: aprendizaje, cálculo, comunidad, gráficas, espacial, pensamiento. 


\begin{abstract}
Evidenced weaknesses in the learning process of differential calculus in the students of Engineering at the Universidad Francisco de Paula Santander Ocaña, we sought the organization of a group of teachers and students in a Community of Learning and a Club of Mathematics, for the use Application software that allowed the strengthening of perception and the graph of thought, key elements for the spatial conception of the environment. The research had a qualitative character and sought to determine the level of spatial thinking of the students of the line of calculation of the engineering programs, and the quantitative thus sought to measure the use of application software. At the conclusion of the project, students were able to improve the graphical representation of two-dimensional functions, using Geogebra, which allows them to solve exercises in less time, besides visualizing them graphically. It was also easier for them to clear their doubts regarding the whole issue of differential calculus presented in the programmatic content of this area. The above allowed to conclude that the spatial thinking of students the ability of the ability to orient in space and achieve to solve the exercises that are presented in the classroom.
\end{abstract}

Keywords: learning, calculation, community, graph, spatial, thought.

\section{INTRODUCCIÓN}

En este artículo se socializan los resultados de la investigación realizada, la cual intentó determinar si el uso del software de aplicación Geogebra contribuye con el fortalecimiento del pensamiento espacial en cálculo diferencial, en los programas de ingeniería mecánica, civil y sistemas ofertados por la Universidad Francisco de Paula Santander Ocaña, a fin de sentar las bases para el posterior diseño de módulos de orientación didáctica relacionados con el uso de este software en temáticas específicas que se trabajen en esta asignatura.

Para el logro de este cometido se constituyó una Comunidad de Aprendizaje, entendida como una comunidad de práctica permanente entre docentes y un Club de Matemáticas con los estudiantes de los programas de ingeniería que tuvieran relación con cálculo diferencial.

En palabras de (Trevisán, 2000) las Comunidades de Aprendizaje o de Práctica fortalece y promueve las interacciones entre los mismos estudiantes y de igual manera entre estudiantes y docentes, además de propiciar situaciones que determinan claramente el proceso de aprendizaje.

Pertenecer a una comunidad de aprendizaje produce en los profesores mayor satisfacción y moral más alta logrando, en consecuencia, reducir el absentismo y la tensión que la tarea docente suele generar (Molina, 2003).

La conformación del Club de Matemáticas se sustenta en el trabajo de investigación sobre comunidades de aprendizaje (Elboj et al., 2002).

En una primera fase se realizó un diagnóstico situacional para establecer el nivel en el que se encontraba el pensamiento espacial en los estudiantes de la línea de cálculo de los programas de ingeniería.

En una segunda fase, la investigación se enfocó en la planeación y desarrollo de una estrategia de aprendizaje sustentada en el uso y apropiación de software de aplicación específico, que permitiera el fortalecimiento del pensamiento espacial con una mirada crítica del entorno y una lectura rigurosa del mismo, para que fuera evidente la aplicación 
del conocimiento matemático por parte del estudiante en la solución de problemas recurrentes del entorno, desde los fundamentos conceptuales adquiridos en cada ingeniería.

En la tercera fase, se evaluaron los resultados en el Club de Matemáticas y se fortaleció la Comunidad de Aprendizaje conformada a partir de una estrategia virtual. Para ello se creó un grupo en la plataforma virtual institucional Moodle con los docentes de cálculo diferencial.

Una de las debilidades evidenciadas en los estudiantes de la línea de cálculo en los programas de ingeniería, es la dificultad para construir y manipular las representaciones mentales de los objetos del espacio, las relaciones entre ellos, sus transformaciones, y por ende, las representaciones materiales de los mismos.

Otra debilidad encontrada radica en que las opciones didácticas que se implementan en el aula, son escasas. Los estudiantes se quejan de que las clases son solo magistrales sin ningún componente que fomente la creatividad $y$ el pensamiento crítico. Manifiestan que las metodologías utilizadas por los docentes, poco potencian su capacidad para representar mentalmente una idea y concretarla en una función específica.

Debido a que el proceso de construcción de conocimiento en cálculo diferencial en los programas de ingeniería, no ha sido el más adecuado, reduciéndose en muchos casos, según las observaciones realizadas por los estudiantes, en la mecanización de conceptos sin ningún sustento práctico; los estudiantes manifiestan tener dificultades para aplicar los conceptos matemáticos en las asignaturas específicas de la carrera o para plantear soluciones creativas a los problemas que se les sugieran.

\section{METODOLOGÍA}

La propuesta se fundamentó en la revisión documental en torno a la conformación de comunidades de aprendizaje, el pensamiento espacial y el uso de software de aplicación para fortalecer el pensamiento espacial en los estudiantes de cálculo diferencial de ingenierías (civil, mecánica y sistemas). La investigación tuvo un carácter cualitativo pues se buscó determinar el nivel de pensamiento espacial de los estudiantes de la línea de cálculo de los programas de ingeniería, y, cuantitativo para medir el uso de software de aplicación; por lo que se elaboraron y realizaron entrevistas a docentes y estudiantes, una encuesta y se está finalizando la fase de consolidación de la comunidad de aprendizaje con la implementación de la estrategia virtual (uso de la plataforma Moodle para el intercambio de conocimientos de pares).

Participaron en la investigación, 20 estudiantes de los programas de ingenierías mecánica, civil y sistemas. Con ellos se conformó el Club de Matemáticas.

Para la selección de los estudiantes se tuvo en cuenta los siguientes criterios: debían ser estudiantes debidamente matriculados en cualquiera de los programas de ingeniería (sistemas, civil o mecánica). Además debían cursar la asignatura de cálculo I (cálculo diferencial) y haber evidenciado en los cursos de nivelación que al inicio de semestre se hacen en todos los programas académicos que oferta la Universidad, un bajo nivel en el análisis gráfico de funciones en dos dimensiones.

La mayoría de estos estudiantes proviene de los municipios de la provincia de Ocaña, que hace parte de la región de El Catatumbo. De los 20 estudiantes que participaron de la experiencia, el $30 \%$ son mujeres y el $70 \%$ 
restante hombres. Sus edades oscilan entre los 15 y 17 años.

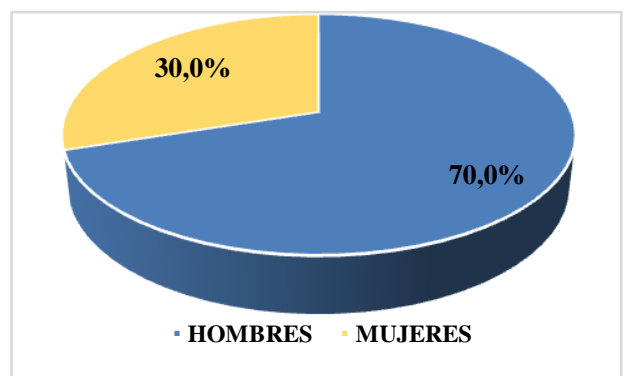

Figura 1. Integrantes del Club de Matemáticas - Género Fuente. Elaboración propia

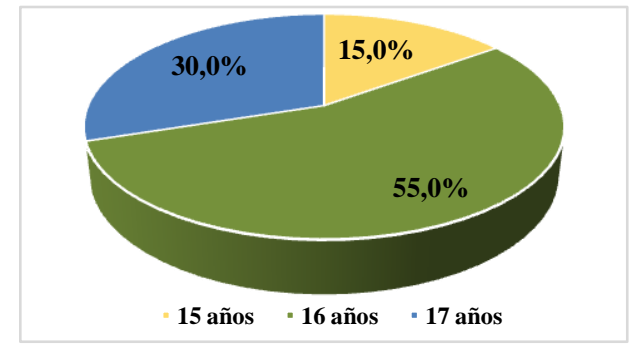

Figura 2. Integrantes del Club de Matemáticas - Edad Fuente. Elaboración propia

En cuanto al desempeño de los estudiantes pertenecientes al Club de Matemáticas durante la fase de aprendizaje del software de aplicación Geogebra, se diseñó e implementó una rúbrica. También se diseñó una entrevista que fue aplicada a los estudiantes finalizado el proceso de capacitación relacionada con el software de aplicación Geogebra. Esta entrevista buscó evidenciar los avances en el proceso de fortalecimiento del pensamiento espacial en ellos.

Para la recolección de los datos se analizó cada una de las respuestas dadas por los estudiantes en la entrevista y se sistematizó el desempeño de cada estudiante durante el proceso de aprendizaje del software de aplicación Geogebra.

En relación con el análisis de datos y posterior sistematización de la información derivada del mismo, se realizó una triangulación con los fundamentos teóricos de la propuesta y el desempeño evidenciado por los estudiantes en el trabajo con Geogebra.

\section{RESULTADOS}

El trabajo realizado durante todo el primer semestre de 2017 permitió observar que los estudiantes que asistieron con frecuencia a los talleres de capacitación a través del Club de Matemáticas mejoraron en la representación gráfica de funciones en dos dimensiones, a partir de la utilización del software Geogebra, pues evidenciaron comprender que con el uso del mismo, fácilmente pueden resolver un ejercicio en menos tiempo, además de visualizarlo gráficamente.

Otro aspecto que se evidenció en la observación de las actividades realizadas por los estudiantes en las sesiones de trabajo promovidas y en las entrevistas que los informantes clave dieron, es el hecho de que a los estudiantes les resultó más fácil despejar sus dudas en relación con cualquier tema de cálculo diferencial que se presentó en el contenido programático de esta área en cada una de las ingenierías.

Los informantes dejaron entrever que al lograr desarrollar con rapidez los ejercicios, pudieron fortalecer la comprensión lógicomatemática de objetos en dos dimensiones, logrando imaginar y visualizar con facilidad una determinada función, comprendiendo su comportamiento y orientación en el espacio.

Esto también se pudo comprobar en la observación del trabajo realizado por los estudiantes y en la valoración de su desempeño con la rúbrica diseñada, pues paulatinamente adquirieron la capacidad de identificar los elementos y herramientas en 
el programa obteniendo así mismo, la solución a los ejercicios que debían resolver.

Por otro lado, la mayoría de los estudiantes entrevistados manifestó que gracias a Geogebra pudieron incrementar su sentido analítico con gran velocidad, desarrollando los ejercicios de una forma muy rápida $\mathrm{y}$ didáctica.

Lo anterior permitió concluir que el pensamiento espacial de los estudiantes que asistieron a las jornadas de formación en el Club de Matemáticas, relacionadas con el aprendizaje del software de aplicación Geogebra, se encuentra en un nivel tal que tienen la capacidad para orientarse en el espacio y lograr resolver los ejercicios que se les presenta en el aula de clase.

En las observaciones que se realizaron en el aula se pudo notar con facilidad que además de fortalecer sus conocimientos matemáticos y de cambiar su visión de los procesos para desarrollar problemas, los estudiantes mostraron un agrado notable por su trabajo matemático basado en el software, actitud que por supuesto mejora en gran medida el aprendizaje ya que pueden realizar procesos como el análisis y la construcción de pensamiento matemático con mayor facilidad.

Se registra en las figuras 1 y 2 como a partir del uso del software de aplicación Geogebra los estudiantes pudieron desarrollar con la herramienta, temas vistos en la clase como: los procesos de la recta real y las funciones y sus cálculos.

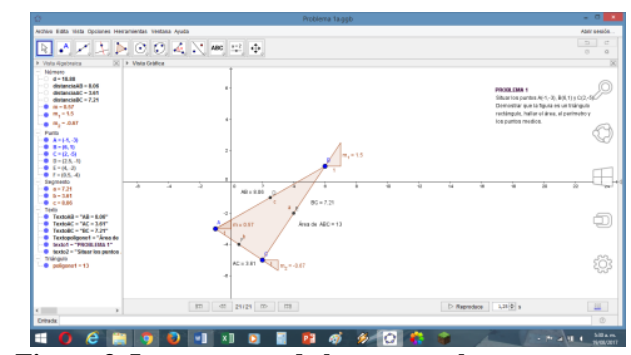

Figura 3. Los procesos de la recta real
Fuente. Elaboración propia

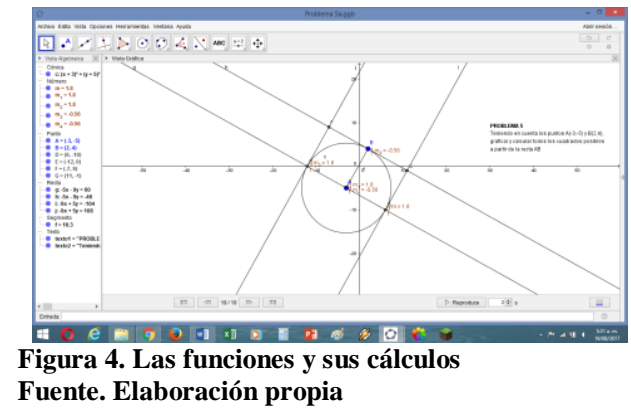

De acuerdo con las actividades realizadas en el Club de Matemáticas a lo largo del semestre, se observó que los estudiantes que asistieron con frecuencia mejoraron en la representación gráfica de funciones en dos dimensiones, a partir de la utilización del software de aplicación Geogebra, pues evidenciaron una mayor comprensión de las temáticas abordadas con el uso del mismo, pudiendo resolver problemas sugeridos en un tiempo menor, además de visualizarlos gráficamente.

Los informantes dejaron entrever que al lograr desarrollar con rapidez los ejercicios, pudieron fortalecer la comprensión lógicomatemática de objetos en dos dimensiones, logrando imaginar y visualizar con facilidad una determinada función, comprendiendo su comportamiento y orientación en el espacio. Esto también se pudo comprobar en la observación del trabajo realizado por los estudiantes, pues paulatinamente adquirieron la capacidad de identificar los elementos y herramientas en el programa obteniendo así mismo, la solución a los ejercicios que debían resolver.

\section{CONCLUSIONES.}

En lo que respecta al objetivo general, se pudo conformar una Comunidad de Aprendizaje con un grupo de docentes y un Club de Matemáticas con los estudiantes interesados, aficionados y acuciosos en el manejo de las matemáticas a través del uso 
de software de aplicación. Para el caso de los estudiantes convocados, previamente se pudo identificar las dificultades que presentaban en relación con el pensamiento espacial en lo referente a la representación gráfica de funciones en dos dimensiones.

La identificación de las dificultades en mención, permitió focalizar el trabajo hacia el uso de software de aplicación Geogebra.

En relación con el primer objetivo específico que buscaba identificar las características fundamentales que evidencia el pensamiento espacial, se realizó una revisión documental que permitió delimitar el trabajo con los estudiantes solo al análisis gráfico de funciones en dos dimensiones a fin de fortalecer la competencia espacial.

En cuanto al segundo objetivo específico que pretendía identificar los elementos clave para la conformación de una Comunidad de Aprendizaje con los docentes y un Club de Matemáticas con los estudiantes de cálculo de los programas de ingeniería, la revisión documental permitió consolidar la estrategia de trabajo a seguir y diseñar las líneas de acción específicas y la estrategia metodológica a implementar con los estudiantes y docentes convocados.

Finalmente, para el caso del tercer objetivo específico que buscaba implementar una estrategia de intervención pedagógica a partir del uso de software de aplicación específico para fortalecer el pensamiento espacial, se decidió trabajar con el software Geogebra. De común acuerdo se establecieron jornadas presenciales de dos horas semanalmente y jornadas virtuales, a través de la plataforma Moodle de acuerdo con las necesidades específicas de los estudiantes.
Para el caso de los docentes, en los diálogos iniciales se logró determinar los aspectos básicos que se debían reforzar en los estudiantes para fortalecer su pensamiento espacial. Para el establecimiento y delimitación de las temáticas a trabajar se partió del consolidado que tanto los planes de estudio de ingeniería como el Departamento de Matemáticas y Física poseen en torno al desempeño de los estudiantes en cada área y la evaluación que estos hacen del desempeño docente.

En relación con las posibilidades que el trabajo realizado con los estudiantes en el Club de Matemáticas sugiere para el desarrollo curricular de la asignatura de cálculo diferencial en los planes de estudio de ingenierías (mecánica, civil y sistemas), es válido afirmar que la incorporación del software de aplicación Geogebra y otros más a esta asignatura, permitirá no solo diversificar las estrategias metodológicas que se empleen en el aula sino también familiarizar a los estudiantes con formas dinámicas de aprendizaje.

\section{FINANCIACIÓN}

Se consolida el Club de Matemáticas como estrategia de aprendizaje de la asignatura cálculo diferencial gracias al proyecto titulado "Comunidad de aprendizaje como estrategia para fortalecer el pensamiento espacial en la línea de cálculo en ingenierías a partir del uso de software de aplicación". El proyecto fue avalado en la convocatoria de la División de Investigación y Extensión UFPSO durante el año 2016 y realizado durante el mismo año.

\section{BIBLIOGRAFÍA}

Aubert, A; Duque, E.; Fisas, M.; Valls, R. (2004). Dialogar y transformar. 
Pedagogía crítica del siglo XXI. Barcelona: Graó.

Campistrous Pérez, Luis La Resolución de Problemas en la Escuela. Pedagogía .1997.

Carretero A. (2001) Metodología didáctica para la enseñanza de Geometría Descriptiva basada en un tutor evaluador y un generador de ejercicios integrados en un entorno de propósito constructivo general.

Cochran-Smith, M. y Lytle, S. (2003). Más allá de la certidumbre: adoptar una actitud indagadora sobre la práctica. En A. Lieberman y L. Miller (Eds.), La indagación como base de la formación del profesorado y la mejora de la educación, pp. 65-80. Barcelona: Octaedro.

Darío, D. O. (1987). Técnicas de Investigación. Santa Fe de Bogotá: Imprenta Nacional.

Díaz, F. H., \& Hernández Díaz, F. (2005). Metodología del estudio. Santa fe de Bogotá: Graw Hill.

Elboj, C., Puigdellívol, I., Soler, M. \& Valls, R. (2002). Comunidades de aprendizaje. Transformar la educación. Barcelona: Graó.

Escudero, J.M. (2009). Comunidades docentes de aprendizaje, formación del profesorado y mejora de la Educación. Ágora para la EF y el Deporte, 10, pp. 7-31.

Estela, M.R.; Saà, J. Cálculo con soporte interactivo en Moodle; Ed. PearsonPrentice Hall; Madrid, 2008.

Flórez, R. (1994). Hacia una pedagogía del conocimiento. Santa fe de Bogotá: McGraw Hill.
Gonzales, A. (1985). Los hábitos de estudio y su relación con el rendimiento académico. Guatemala.

Gómez, Flores y otros, (1996). Metodología de la investigación cualitativa.

Goode, w. y. (1984). Métodos de investigación social. México: Trillas.

Hord, S. (1997) Professional Learning Communities: What are they and why are they important? Issues... About Change, 6(1), Disponible en de http://www.sedl.org/change/issues/issues61. $\underline{\mathrm{html}}$

Ibrain, L. P. (s.f.). Construcción de una escala Grafica. CIN CEL.

Julio, A. B. (2014). Temas selectos de organización educativa. Bonobos Editores S.

Maddox, H. (1988). Como estudiar. Santa fe de Bogotá: Printer Colombia S A.

Molina, E. (2005) Creación y desarrollo de comunidades de aprendizaje: hacia la mejora educativa. Revista de Educación, 337, pp. 235-250

Murillo, F.J. (2002). La "Mejora de la Escuela": concepto y caracterización. En F.J. Murillo y M. Muñoz-Repiso (Coords.), La mejora de la escuela: un cambio de mirada. Barcelona: Octaedro.

Obiols, G. (2007). Como estudiar. Buenos Aires: Plus Ultra.

Oedro, L. (1967). Esquemas de organización de las unidades de aprendizaje. San Luis: Cin Cel. 